\title{
Surface IgM-Inducing Factor in the Culture Supernatant of Bursal Epithelial Cells Derived from Chick Embryos.
}

\author{
Song Han, Mineyuki Kondo, Asaki Abe, Yoshinobu Kimura, \\ Kazumasa Ohtsuki and Yasuhiro Kondo \\ Faculty of Agriculture, Okayama University, Okayama 700-8530, Japan
}

\begin{abstract}
Bursal epithelial cells derived from 14-day-old chick embryos were cultured under a serum-free condition and the recovered culture supernatant was analyzed for surface immunoglobulin M (sIgM)-inducing activity on the bursacytes of 12-day-old chick embryos. Surface IgM positive rate of bursacytes incubated with the culture supernatant increased significantly, thus indicating the presence of sIgM-inducing factor(s) in the culture supernatant. The sIgM-inducing factor(s) in the supernatant was analyzed by gel filtration chromatography and HPLC. Results from these analyses indicated that the factor may be a polypeptide with a molecular weight of about 1300. This substance which is different from already known factors, such as Bursin, may be a newly discovered B cell differentiating factor in the bursa of Fabricius that promotes chicken B cell differentiation.
\end{abstract}

Key words : bursa of Fabricius, epithelial cells, sIgM-inducing factor

\section{Introduction}

It has been well documented that the bursa of Fabricius functions as a Blymphopoietic organ in avian species. Lymphoid follicles present in the lamina propria of the bursa are composed of lymphoid cells and a network of reticuloepithelial cells (Péault et al., 1987). Extrinsic lymphoid precursor cells colonize the follicles of chick embryos between 8 and 14 days of embryogenesis. The colonized cells differentiate and proliferate under the microenvironment presented by follicular reticuloepithelial cells of the bursa (Péault et al., 1987).

Bursa-derived humoral factors involved in B cell development have been reported in chickens. Bursal extract induced Bu-1 alloantigen expression on the chicken $\mathrm{B}$ cell surface (Brand and Gilmour, 1976). Audhya et al. (1986) isolated a bursa-derived low molecular weight peptide, which elevated cAMP level in human B lymphoma cell line and termed it Bursin. Bursin also acted on expression of Bu-1 antigen on bursacytes, diversification of immunoglobulin (Otsubo et al., 2001). Other factors have been reported in the bursa. For example, bursal extract induced Bu-1 alloantigen expression on the chicken B cell surface (Brand and Gilmour, 1976). Serum-supplemented culture supernatant of bursal epithelium elevated the Ia antigen-positive rate of chicken B cells (Eerola et al., 1982). However, unlike Bursin, their chemical nature has not been

Received : September 27, 2002, Accepted : January 6, 2003

Corresponding Author: Song Han 
determined. Since the serum present in culture media used for bursal cell culture makes the biochemical analysis of factors in the supernatant difficult, serum-free culture of bursal epithelium has been done (Han et al., 2003). In the present study, we analyzed the factor which induces immunoglobulin M (IgM) expression on surface of immature chick embryos bursacyte by gel filtration chromatography and HPLC systems.

\section{Materials and Methods}

Preparation of culture supernatant : Supernatants were prepared from primary cultures of bursal epithelial cells collected from 14-day-old chick embryos of specificpathogen-free (SPF) White Leghorn Shaver Strain hen as previously reported (Han et al., 2003). Briefly, the bursae were removed from 14-day-old chick embryos. They were cut into small pieces and dispersed to single cells by incubating with $0.2 \%$ collagenase (Sigma Chemical Co., USA). After washing, the cells $\left(1.5 \times 10^{6}\right.$ cells $)$ were resuspended in $1.5 \mathrm{~m} l$ of serum-free DMEM/Ham's F12 1: 1 mixture medium (Sigma) supplemented with $10^{-8} \mathrm{~g} / \mathrm{ml}$ of epidermal growth factor (Sigma Chemical Co., USA), $10^{-4} \mathrm{~g} / \mathrm{m} l$ of insulin (Sigma), $10^{-5} \mathrm{~g} / \mathrm{m} l$ of transferrin (Sigma), $4.32 \times 10^{-9} \mathrm{~g} / \mathrm{m} l$ of sodiumselenite (Sigma), $10^{-7} \mathrm{~g} / \mathrm{m} l$ of hydrocortisone (Sigma) and $1.22 \times 10^{-6} \mathrm{~g} / \mathrm{m} l$ of 2-aminoethanol (Sigma) and seeded on a collagen coated 12-well plastic plate. The plate was incubated at $39^{\circ} \mathrm{C}$ under $5 \% \mathrm{CO}_{2}$ in air. Culture medium was changed every 3 days. Monolayers composed of keratin-positive polygonal epithelial cells were maintained until 5 months without any apparent morphological abnormalities (Han et al., 2003). After 3 weeks, the supernatant was recovered from the well, and centrifuged at $350 \times \mathrm{g}$ for $10 \mathrm{~min}$ to give a supernatant, which was used in subsequent analyses, and sediment which was discarded. The supernatant was concentrated and then analyzed by gel filtration and HPLC.

Counting of sIgM positive cells in the bursacytes : The bursae were collected from chick embryos at day 12 of embryogenesis. They were cut into small pieces with small scissors in the culture medium. The cells suspended in medium were collected and used as bursal lymphoid cells (bursacytes). Purity if the lymphoid cells in the cell suspension, determined by Giemsa staining were above $85 \%$. The cells were incubated with the culture supernatant of bursal epithelium (BECS) or fraction obtained from BECS as described later for 0 to 96 hours at $39^{\circ} \mathrm{C}$ under $5 \% \mathrm{CO}_{2}$ in air. After incubation, the number of sIgM positive cells were counted by indirect immunofluorescence using goat anti-chicken $\mu$ chain antibody (Bethyl Laboratory Inc., USA) and rabbit anti-goat IgG-FITC conjugated antibody (Vector Laboratories, Inc., USA). Surface IgM positive rates were calculated in bursacytes treated with the BECS and fractions of gel filtration and HPLC.

Fractionation of the supernatant by Sephadex G-50 : BECS was concentrated 10 -fold by evaporation and fractionated on Sephadex G-50 (Pharmacia Fine Chemicals, Sweden) column $(2 \times 30 \mathrm{~cm})$ eluted with Borate Buffer Solution $(0.16 \mathrm{M}, \mathrm{pH} 8.2)$. Elution was monitored by absorbance at $215 \mathrm{~nm}$ and $280 \mathrm{~nm}$ using a Shimadzu UV-14002 spectrophotometer. Two fractions, fraction I (eluted between $108 \mathrm{~min}$ and $168 \mathrm{~min}$ ) and fraction II (eluted between $210 \mathrm{~min}$ and $216 \mathrm{~min}$ ), were obtained. These fractions 
were recovered, concentrated 10-fold by evaporation and subjected to detection of sIgMinducing activity. The lower molecular fraction (fraction II) was found to be active.

Fractionation by reversed-phase HPLC : Fraction II was further fractionated by reversed-phase HPLC on a Capcell PAK C18 column $(10 \mathrm{~mm} \times 250 \mathrm{~mm}$, Shiseido, Japan) eluted with trifluoroacetic acid-aceto nitrile (Eluent A : 0.05\% TFA ; Eluent $\mathrm{B}: \mathrm{TFA}+80 \% \mathrm{CH}_{3} \mathrm{CN}$ ) solution at a flow rate of $1.5 \mathrm{ml} / \mathrm{min}$. Elution was monitored by absorbance at $280 \mathrm{~nm}$ using a UV-vis detector (Shimadzu; SPD-10A). Three fractions, fraction I (eluted between $27 \mathrm{~min}$ and $29 \mathrm{~min}$ ), fraction II (eluted between 36 min and $38 \mathrm{~min}$ ) and fraction III (eluted between $45 \mathrm{~min}$ to $47 \mathrm{~min}$ ), were obtained. After a 10-fold concentration and dialysis against phosphate buffer solution (PBS, $\mathrm{pH}$ 7.4, $0.01 \mathrm{M}$ ), they were subjected to detection of sIgM-inducing activity.

Fractionation by gel filtration-HPLC : The third fraction eluted by reversed-phase HPLC was found to be active and further fractionated by analytical gel filtration-HPLC (Superdex Peptide HR10/30, Pharmacia) eluted with PBS (pH 7.4, 0.01 M) at a flow rate of $0.25 \mathrm{ml} / \mathrm{min}$. Elution was monitored by absorbance at $215 \mathrm{~nm}$ using a UV-vis detector. Two fractions, fraction I (eluted between $44 \mathrm{~min}$ to $48 \mathrm{~min}$ ) and fraction II (eluted between $57 \mathrm{~min}$ and $63 \mathrm{~min}$ ), were obtained. The fractions were concentrated 10-fold by evaporation and subjected to detection of sIgM-inducing activity.

Molecular weight estimation of sIgM-inducing factor : In order to estimate the molecular weight of the sIgM-inducing factor in culture supernatant of bursal epithelium, elution time of the factor was compared with those of glycopeptide (MW : 2576), leucine-glycine (MW : 206.25) and glycine (MW : 75.07) using a gel filtration column HPLC (Superdex Peptide HR10/30) eluted with PBS (0.01 M, pH 7.4). Fractions were detected by absorbance at $215 \mathrm{~nm}$.

Statistical analysis : Analysis of variance was used to determine significances in sIgM positive rates between bursacytes incubated with culture medium and bursacytes incubated with the BECS or fractions of BECS. All significances were determined at the 5\% level. All results are given as means and standard errors.

\section{Results}

Surface IgM inducing activity of bursal epithelium culture supernatant : Surface IgM positive cells were observed in bursacytes collected from 12-day-old chick embryos just as in a previous report (Grossi et al., 1977). As shown in Fig. 1, the positive rate was not so high at 0 hour $(13.6 \pm 1.7 \%$ : Mean $\pm \mathrm{SE})$. Positive rates tended to be increased by incubating with culture supernatant of bursal epithelium for 96 hours (31.3 $\pm 8.7 \%)$, but the rates did not change by incubating with culture medium (18.0 \pm $3.6 \%$ : incubation for 96 hours), suggesting that the factor(s) promoting sIgM expression on the bursacytes of chick embryo are present in the supernatant (Fig. 1).

Surface IgM inducing activity of Sephadex G-50-fractions of supernatant : Figure 2-A shows the fraction pattern of bursal epithelium culture supernatant by Sephadex G50. Two fractions were separated at $215 \mathrm{~nm}$ and $280 \mathrm{~nm}$. The higher molecular fraction (fraction I) and lower molecular fraction (fraction II) were monitored for their activity in inducing sIgM expression on bursacytes of 12-day-old chick embryos as described 
above. Surface IgM positive rates of bursacytes were increased by incubating with the lower molecular fraction ( $\mathrm{p}<0.05$ in cells incubated for 48 hours), but the rates did not change by incubating with the higher molecular fraction (Fig. 2-B).

Surface IgM inducing activity of fractions separated by reversed-phase HPLC : The lower molecular fraction separated by Sephadex G-50 was then fractionated by reversed-phase HPLC. Figure 3-A shows its elution pattern. Three fractions (fraction I, fraction II and fraction III) were separated. Surface IgM positive rates of bursacytes

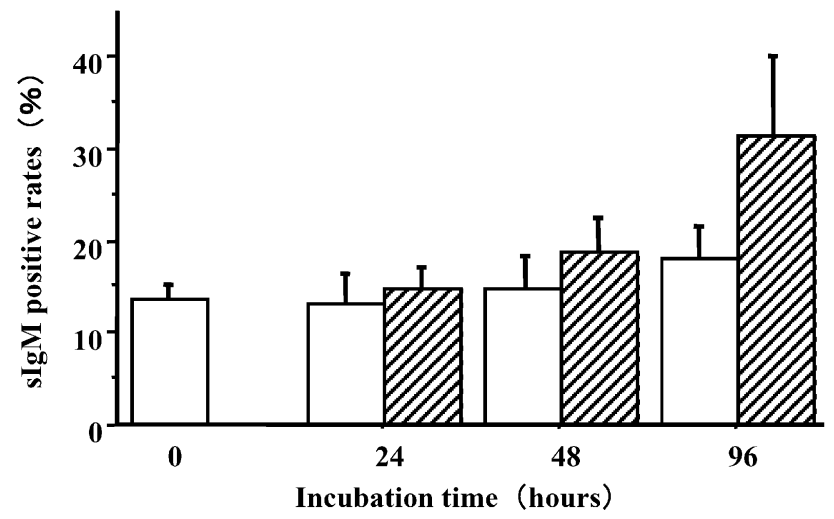

Fig. 1. Surface IgM positive rates of bursacytes incubated with bursal epithelium culture supernatant (BECS). Open columns represent the positive rates of cells incubated with culture medium (control), while shadowed ones represent the positive rates of cells incubated with BECS.

A

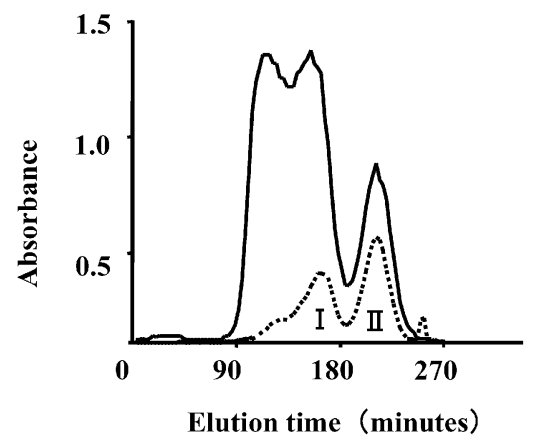

B

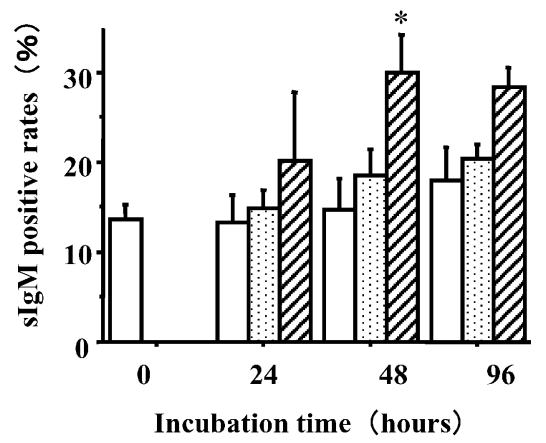

Fig. 2. (A) Sephadex G-50 fractionation pattern of fraction patterns of bursal epithelial cell-culture supernatant. Solid line and dotted line represent absorbance at $215 \mathrm{~nm}$ and $280 \mathrm{~nm}$, respectively. (B) The sIgM positive rates of bursacytes incubated with the fraction. Open columns, dotted columns and shadowed columns represent the sIgM positive rates of cells incubated with culture medium, fractions I and II obtained by gel filtration chromatography of BECS using Sephadex G-50 column, respectively. $*=$ Significantly different from mean of the control $(\mathrm{P}<0.05)$. 
incubated with fractions III for 24 to 96 hours were significantly higher than those incubated with culture medium $(\mathrm{p}<0.01)$. However, the positive rates of bursacytes did not change by incubating with fractions I and II (Fig. 3-B).

Surface IgM inducing activity of fractions separated by gel filtration-HPLC : The active fraction separated by reversed-phase HPLC was further fractionated by gel filtration-HPLC system. As shown in Fig. 4-A, two fractions were separated. The First fraction showed sIgM inducing activity after 24, 48 and 96 hours of incubation ( $\mathrm{p}$ $<0.05$ ), but the second fraction was not active (Fig. 4-B). The fraction pattern and presence of sIgM inducing activity in fractions indicated that the activity may be due to a single factor in the bursal epithelium culture supernatant.

Molecular weight of the sIgM-inducing factor: When three molecular weight markers, glycopeptide, leucine-glycine and glycine were subjected to separation with the gel filtration-HPLC system used in fractionation of the sIgM-inducing factor, the glycopeptide, leucine-glycine and glycine were eluted after 42 minutes, 65 minutes and 70 minutes, respectively (Fig. 5-A). Figure 5-B shows the relationship between the elution volumes $(\mathrm{m} l)$ of the three molecular weight markers and the logarithm to the base ten of their molecular weights. A straight line was drawn from the elution volumes and the logarithms of molecular weights of the three molecular weight markers. From the straight line, corresponding to the elution time (48 min)(Fig. 4-A) of the active fraction obtained by gel filtration-HPLC was about 3.118. Thus, the molecular weight of the first fraction (sIgM inducing factor) can be deduced to be approximately 1300 .

$\mathbf{A}$

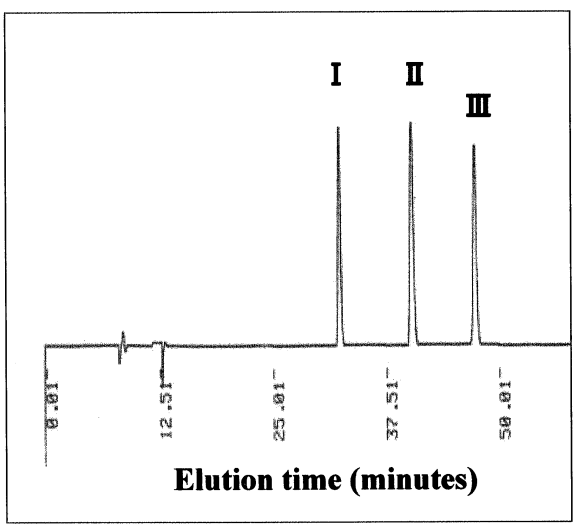

B

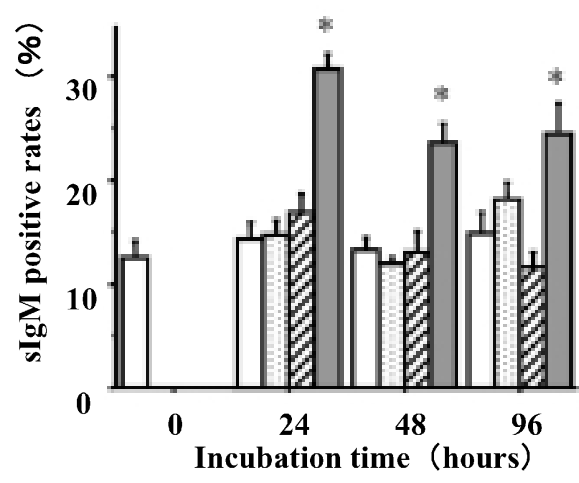

Fig. 3. (A) Elution pattern of Sephadex G-50-low molecular fraction by reverse-phase HPLC and (B) SIgM positive rates of the bursacytes incubated with culture medium (open columns), fraction I (dotted columns), II (shadowed columns) and III (black columns) obtained by separation of low molecular fraction of Sephadex G-50 using reversed-phase HPLC, respectively. ${ }^{*}=$ Significantly different from means of the controls $(\mathrm{P}$ $<0.05)$. 
$\mathbf{A}$

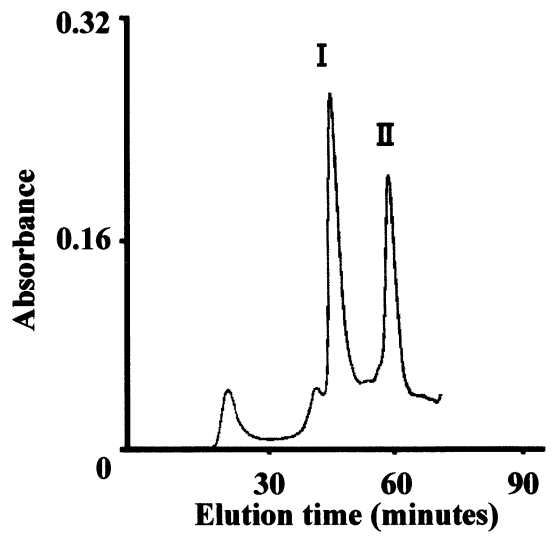

B

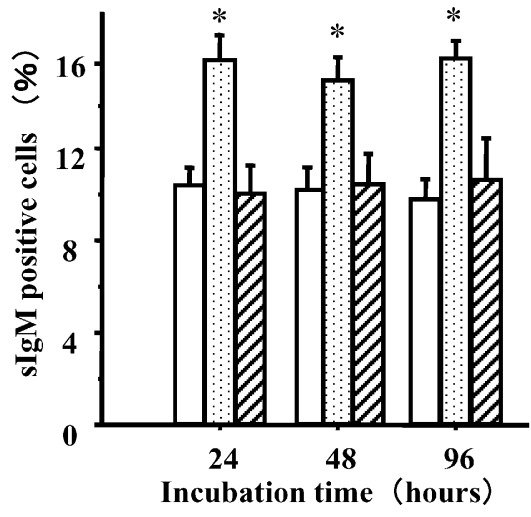

Fig. 4. (A) Elution pattern of reversed-phase HPLC-peak III fraction by gel filtration HPLC and (B) isolated by reversed-phase HPLC (A) and SIgM positive rates of the bursacytes incubated with culture medium (open columns), fraction I (dotted columns) and II (shadowed columns) obtained by separation of fraction III of reversed-phase HPLC using gel filtration-HPLC, respectively. $*=$ Significantly different from the means of controls $(\mathrm{P}<0.01)$.

$\mathbf{A}$

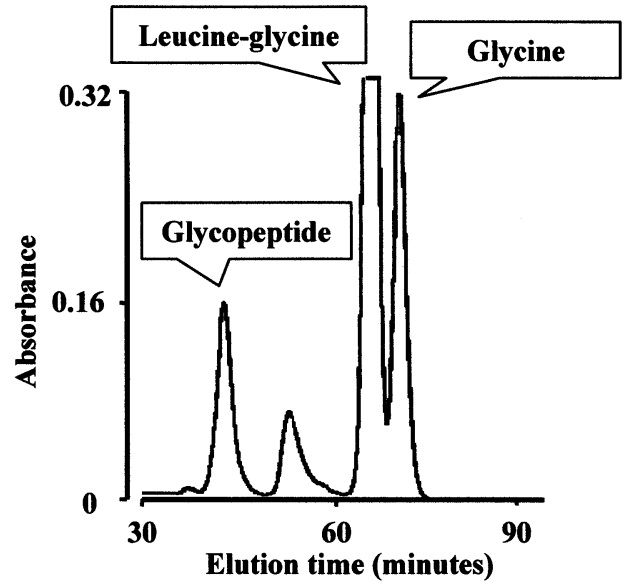

B

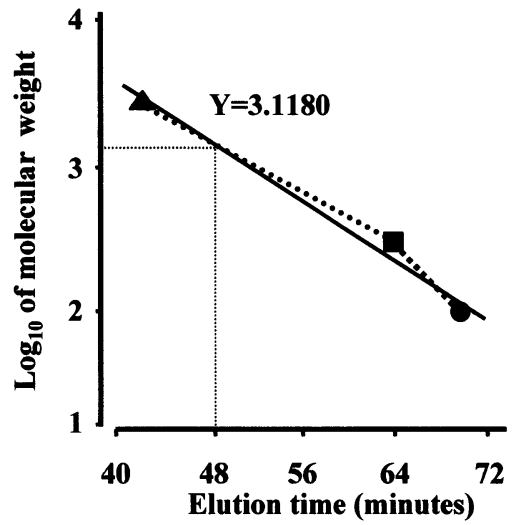

Fig. 5. (A) Elution times of molecular weight markers by gel filtration-HPLC and (B) relations between elution times of the markers (glycopeptide : $\boldsymbol{O}$, leucine-glycine : $\boldsymbol{\Delta}$ and glycine : and fraction II separated by gel filtration-HPLC which is presented by dotted line, and $\log 10$ of their molecular weights. $\mathrm{Y}=3.1180$ in $\mathrm{B}$ indicates $\mathrm{y}$-coordinate of peak. 


\section{Discussion}

The bursa of Fabricius is known to be essential for the generation of diversified B cell repertoires in chicken, and reticuloepithelial cells in the bursal lymphoid follicles play essential roles in B cell diversification. Extrinsic B cell precursors have been reported to enter and colonize the lymphoid follicles of the bursa of chick embryos at 8 to 14 days of embryonation (Péault et al., 1987). Appearance of B cell diversity in response to non-self antigens and deletion of $\mathrm{B}$ cell populations responsible for elimination of self-antigens occur in the bursal lymphoid follicles under the microenvironment presented by bursal non-lymphoid cells, especially, reticuloepithelial cells (Boyd et al., 1983). In this process, a rearrangement of antibody genes occurs in the B lymphoid cells which have settled in the bursal lymphoid follicles of chick embryos, and B cell clones specific in their antibody genes appear (Pink et al., 1985 ; Ratcliff et al., 1985 ; Pink and Lassila, 1987 ; Sayegh et al., 2000). Subsequently, immunoglobulin molecules, synthesized according to the rearranged gene express on membrane of bursal lymphoid cells. In chick embryos, IgM molecules are reported to be first expressed on bursal lymphoid cells at day 12 of embryogenesis (Grossi et al., 1977). These results indicate that antibody gene rearrangement starts in the bursacytes of chick embryos between days 8 to 12 of embryogenesis. In the present study, surface IgM (sIgM) positive rates of bursacytes collected from 12-day-old chick embryos were elevated when the cells were incubated with BECS, suggesting the presence of sIgM-inducing factor(s) in the culture supernatant. In mammals, the appearance of cytoplasmic $\mu$ chain with a surrogate light chain precedes expression of surface $\mu$ chain during embryonic B cell development in the bone marrow (Abbas et al., 1997). On the other hand, surface and cytoplasmic IgM (cIgM) have been reported to be expressed simultaneously during embryonic B cell development in the bursa (Grossi et al., 1977). Expression of sIgM on B lymphoid cells is thought to be the critical point of B cell differentiation in the bursa, because synthesis of $\mu$ chain and simultaneous expression of sIg and cIg followed the rearrangement of Ig genes in B lymphoid cells. Accordingly, discovery of sIgM-inducing factor(s) in the BECS is significant in the study of avian immunology. There may be a possibility that elevation of the sIgM positive rate is due to augmentation of Ig gene rearrangement in the bursacytes that are applied with the bursal epithelium culture supernatant.

The significance of reticuloepithelial cells in the lymphoid follicles of the bursa in B cell proliferation and differentiation has already been established (Boyd et al., 1983). Reconstitution studies using the cyclophosphamide model of immunodeficiency clearly indicate the importance of the bursal epithelium in B cell differentiation (Toivanen et al., $1972 \mathrm{a}, 1972 \mathrm{~b}$ ). Eerola et al. (1982) demonstrated Ia antigen inducing activity on B cell surfaces using serum-supplemented culture from bursal epithelial cells. Also, factors involved in B cell development have been demonstrated by bursal organ culture (Nowak et al., 1990) and in bursal extracts (Brand et al., 1976). Among these factors, a bursa-derived factor (termed as Bursin) that induces elevation of cAMP levels of a human B lymphoma cell line has been reported to be a tripeptide whose molecular 
weight was about 376 (Audhya et al., 1986).

In the present study, sIgM inducing activity in BECS was attributed to a factor in the supernatant which was isolated by gel filtration and HPLC system, and weight of the factor was estimated at about 1300 . The factor reported in the present study is considered to be newly discovered substance different from Bursin reported by Audhya et al. (1986), although the sizes of other factors involved in B cell development are unclear. We consider that like Bursin, this compound might also be a peptide because it had UV absorption at $215 \mathrm{~nm}$ (all peptide have this characteristics). However, it may be possible that the factor is a substance other than a peptide.

Recently, Otsubo et al. (2001) reported that bursin may act on the appearance of IgM positive cells during development of the bursa because anti-bursin antibody suppressed the appearance of IgM positive cells in the embryonic bursa. In the present study, Bursin activity could not be demonstrated in BCEM. Reason explaining this difference is unclear. However, it may be postulated that Bursin activity seemed to be lost during fractionation because its activity is lower than the sIgM-inducing activity reported in the present study.

We conclude that bursal epithelial cells produce a factor inducing sIgM expression on B lymphoid cells in the bursa of chick embryo, and the factor may be a low molecular peptide with a molecular weight of about 1300. Further investigation about the factor must be done in respect to the detail biochemical features, especially amino acid composition and sequences on the assumption that the factor is a peptide.

\section{References}

Abbas AK, Lichtman AH and Pober JS. Maturation of B lymphocytes and expression of immunoglobulin genes. In : Cellular and Molecular Immunology. pp. 66-95. W.B. Saunders Company. Philadelphia. 1997.

Audhya T, Kroon D, Heavner G, Viamontes G and Goldstein G. Tripeptide structure of bursin, a selective B-cell differentiating hormone of the bursa of Fabricius. Science, 231 : 997-999. 1986.

Boyd RL, Ward HA and Muller HK. Bursal and thymic reticular epithelial cells in the chicken : induction of B- and T-lymphocyte differentiation by in vitro monolayer cultures. Journal of the Reticuloendothelial Society, 34 : 383-393. 1983.

Brand A, Gilmour DG and Goldstein G. Lymphocyte-differentiating hormone of bursa of Fabricius. Science, 193 : 319-321. 1976.

Eerola E, Lassila O, Gilmour DG and Toivanen A. Induction of B cell differentiation in vitro by bursal epithelium. Journal of Immunology, $128: 2652-2655.1982$.

Grossi CH, Lydyard PM and Cooper MD. Ontogeny of B cells in the chicken. II. Changing patterns of cytoplasmic IgM expression and modulation requirements for surface IgM by anti- $\mu$ antibodies. Journal of Immunology, 119 : 749-756, 1977.

Han S, Mitsusada E, Abe A and Kondo Y. Serum-free culture of chicken bursal epithelial cells. Japanese Poultry Science, 40 : 69-73, 2003.

Nowak SJ, Vainio O and Lassila O. In vitro organ culture of embryonic bursa of Fabricius. Developmental and Comparative Immunology, 14 : 239-246. 1990.

Otsubo Y, Chen N, Kajiwara E, Horiuchi H, Matsuda H and Furusawa S. Role of bursin in the development of B lymphocytes in chicken embryonic bursa of Fabricius. Developmental \& Comparative Immunology, 25 : 485-493. 2001.

Péault B, D-Lievre F and Le Douarin NM. Cellular interaction occurring during primary lymphoid organ ontogeny in birds. In : Avian Immunology : Basis and Practice (Toivanen 
A and Toivanen P eds). Vol. 1. pp. 39-63. CRC Press. Boca Raton. 1987.

Pink JR, Vainio O and Rijnbeek A-M. Clones of B lymphocytes in individual follicles of the bursa of Fabricius. European Journal of Immunology, 15 : 83-87. 1985.

Pink JR and Lassila O. B-cell commitment and diversification in the bursa of Fabricius. Current topics in Immunology, $135: 57-64.1987$.

Ratcliffe MJH. The ontogeny and cloning of B cells in the bursa of Fabricius. Immunology Today, $6: 223-231.1985$.

Sayegh CE, Demaries SL, Pike KA, Friedman JE and Ratcliffe MJH. The chicken B-cell receptor complex and its role in avian B-cell development. Immunological Reviews, $175:$ 187-200. 2000.

Toivanen P, Toivanen A and Good RA. Ontogeny of bursal function in the chicken. I. Embryonic stem cell for humoral immunity. Journal of Immunology, 109 : 1058-1070. 1972 a.

Toivanen P, Toivanen A and Good RA. Ontogeny of bursal function in the chicken. III. Immunocompetent cell for humoral immunity. Journal of Experimental Medicine, 136 : 816-831. 1972 b. 